\title{
Gene sequences useful for predicting relatedness of whole genomes in bacteria
}

\author{
Daniel R. Zeigler \\ Bacillus Genetic Stock Center, Department of Biochemistry, The Ohio State University, \\ Columbus, $\mathrm{OH}$ 43210, USA
}

Correspondence

Daniel R. Zeigler

zeigler.1@osu.edu

\begin{abstract}
Thirty-two protein-encoding genes that are distributed widely among bacterial genomes were tested for the potential usefulness of their DNA sequences in assigning bacterial strains to species. From publicly available data, it was possible to make 49 pairwise comparisons of whole bacterial genomes that were related at the genus or subgenus level. DNA sequence identity scores for eight of the genes correlated strongly with overall sequence identity scores for the genome pairs. Even single-gene alignments could predict overall genome relatedness with a high degree of precision and accuracy. Predictions could be refined further by including two or three genes in the analysis. The proposal that sequence analysis of a small set of protein-encoding genes could reliably assign novel strains or isolates to bacterial species is strongly supported.
\end{abstract}

\section{INTRODUCTION}

The current concept of the bacterial species - a 'genomically coherent' group of strains that share many common traits (Rosselló-Mora \& Amann, 2001) - rests squarely on the availability of reliable techniques to quantify the relatedness of bacterial genomes. Although many such techniques exist [for a recent review, see Gürtler \& Mayall (2001)], analysis of DNA-DNA hybridization values remains the 'gold standard' for defining bacterial species (Wayne et al., 1987; Stackebrandt et al., 2002). Techniques required to obtain these values, however, tend to be expensive and timeconsuming and often require specialized instruments or radioactive labels (Johnson, 1994). Furthermore, as many parameters affect DNA-DNA reassociation, hybridization values may be difficult to reproduce in different laboratories.

An alternative approach to quantification of genome relatedness is to compare selected DNA sequences for a group of bacterial strains. The core technology for this method, DNA sequencing, is relatively rapid and inexpensive, highly reproducible and readily available to virtually any research group through specialized sequencing centres. Databases of gene sequences and computer applications to compare them are, likewise, freely available. For these reasons, DNA sequence analysis has taken an increasingly important role in taxonomic studies in recent years.

The bacterial species concept, however, requires that entire

Published online ahead of print on 9 May 2003 as DOI 10.1099/ ijs.0.02713-0.

A spreadsheet listing sequence identity scores for all candidates with each genome comparison is available as supplementary data in IJSEM Online. genomes are compared. If sequence analysis is to augment, or even replace, DNA-DNA hybridization in defining species, it is paramount that taxonomists identify genes that can represent whole genomes reliably for the purposes of comparison. Recently, an ad hoc committee for the re-evaluation of the species definition in bacteria issued a call for identification of a set of such genes (Stackebrandt et al., 2002). The committee's consensus was that analysis of at least five genes of diverse chromosomal loci and wide distribution could provide sufficient information to distinguish a bacterial species from related taxa. Once a species was defined in this way, sequence information from a single member of this gene set may be enough to assign additional strains to the species (Stackebrandt et al., 2002).

It is an open question how much information any given gene sequence can provide about the genome that contains it. Sequence differences between related organisms within a given gene are presumably due to slow, continual acquisition of random mutations, which are subject to selection and inherited vertically. Differences seen in whole-genome comparisons, however, are the sum of this vertical inheritance and any number of horizontal transfer events that involve simultaneous acquisition of many genes through transformation, conjugation or bacteriophage infection. Genome reduction through gene inactivation and deletion further complicates the picture. The relative rates of these factors - random mutation, horizontal transfer and genome reduction - are poorly understood.

The goal of the current study is to obtain statistical evidence that individual gene sequences diverge at a rate that reflects the overall rate of genome divergence and to identify genes that could best serve as predictors of genome relatedness. Publicly available bacterial genome sequences were used to 
identify over 30 genes that satisfy the ad hoc committee's criteria (Stackebrandt et al., 2002). When closely related bacteria were compared, the frequency of identical residues in individual gene alignments correlated with the frequency of identical residues in whole-genome alignments with $R^{2} \geqslant 0.9$ for each of eight genes from this set. The highestscoring sequence from the set, $r e c N$, could be used to predict whole-genome relatedness with high accuracy for a test set of 44 bacterial genomes. Combining data from two or three genes could further refine this prediction. It appears that a small number of carefully selected gene sequences can indeed equal, or perhaps even surpass, the precision of DNA-DNA hybridization for quantification of genome relatedness.

\section{METHODS}

Genomic sequence data. All genomic sequences analysed in this study were obtained from publicly available databases. GenBank accession numbers for the complete but unannotated Neisseria gonorrhoeae and Bacillus anthracis genomes are AE004969 (available at $\mathrm{ftp}: / / \mathrm{ftp} . g e n o m e . o u . e d u / p u b / g o n o /)$ and AAAC01000001, respectively. Sequences for Bordetella bronchiseptica RB50, Bordetella parapertussis 12822, Bordetella pertussis Tahoma 1, Chlamydophila abortus, Corynebacterium diphtheriae NCTC 13129, Mycobacterium bovis AF2122/97, Neisseria meningitidis FAM18 and Yersinia enterocolitica 8081 were obtained from the Sanger Institute microbial genomes site (http://www.sanger.ac.uk/Projects/Microbes/). All other sequences were downloaded from the NCBI microbial genomes site (http://www.ncbi.nlm.nih.gov/PMGifs/Genomes/micr.html). Individual gene sequences were obtained from the genomic sequences, following confirmation through BLAST analysis of their uniqueness within the genome and their high sequence similarity $\left(P<10^{-6}\right)$ to their Escherichia coli K-12 and Bacillus subtilis 168 orthologues.

Individual gene and whole-genome alignments. For calculating DNA sequence identity for individual genes, sequences obtained from related organisms were aligned with CLUSTAL $\mathrm{W}$ and a distance matrix was computed (Thompson et al., 1994). Pairs of whole genomes were aligned by using the NUCMER application (Delcher et al., 2002) with the following parameters: breakLen $=500$, minCluster $=40$, $\operatorname{diagFactor}=0 \cdot 15, \operatorname{maxGap}=250$ and $\operatorname{minMatch}=12$. To ensure that the algorithm found all possible alignments, each pair was analysed twice with the reference and query files swapped. The resulting output files, giving the coordinates of regions of sequence similarity between the two genomes, were combined and duplicate regions were removed from the list. When two neighbouring regions shared overlapping end-points, the common segment was divided equally between them. Two similarity estimates were calculated from each genomic sequence comparison. DNA sequence identity for conserved regions was calculated as the mean sequence identity of the homologous regions, weighted by each region's length in nucleotides. DNA sequence identity for a pair of whole genomes was calculated by multiplying the sequence identity of their conserved regions by the ratio of the net length of the conserved regions to the mean length of the two genomes.

Statistical analysis. Univariate linear regression models were used to assess the predictive ability of sequence identities for each individual gene with respect to sequence identities for whole-genome alignments. Step-wise linear regression procedures were used to find the subset of genes that best predicted the whole-genome alignment. Prediction intervals were calculated and the upper/lower limits of these intervals were used to determine the cut-point for the desired $70 \%$ alignment.

\section{RESULTS AND DISCUSSION}

\section{DNA sequence identity between related bacterial genomes}

Among publicly available bacterial genome sequences are several groups of organisms that are related at the genus or species level. At the beginning of this study (in July 2002), 44 genomes that could be grouped into 16 genera were identified (Table 1). It was known that the genera Escherichia, Salmonella and Shigella were highly related (Brenner et al., 1969, 1972; Crosa et al., 1973), so they were treated as if they belonged to a single genus. Genome sequences within each of the 16 groups were aligned in pairwise fashion by the NUCMER application (Delcher et al., 2002) as described in Methods. These alignments identified two sets of sequences, those conserved between both genomes and those unique to each genome. The first set would primarily include elements of the ancestral genome that have been inherited vertically by both species. DNA sequence identity of these conserved regions can be quantified (Table 1), providing an index of the degree of genome divergence that can be explained by random mutation and selection. DNA sequence identity for whole-genome sequences can also be quantified to measure divergence due to all processes, including vertical and horizontal transfer and genome reduction (Table 1).

It is encouraging that the whole-genome sequence identity figures calculated in this study correlate well with available genome similarity measurements obtained by DNA-DNA hybridization. For example, Brenner et al. (1972) and Crosa et al. (1973) obtained similarity estimates for Salmonella typhimurium with Salmonella typhi (88\%), E. coli (45$46 \%)$ and Shigella flexneri (38-39\%) and for E. coli with Shigella flexneri (84\%). Among the Chlamydiaceae, similarity estimates for Chlamydia muridarum with Chlamydia trachomatis (65\%) (Weiss et al., 1970) and among various strains of Chlamydophila pneumoniae (94-100 \%) (Fukushi \& Hirai, 1989) were also comparable with the estimates in this study. Finally, Somerville \& Jones (1972) measured no detectable competition between Bacillus subtilis and Bacillus anthracis genomic DNA under conditions in which Bacillus anthracis competed with other members of the Bacillus cereus group at levels from 59 to $100 \%$. Whilst these hybridization studies were conducted by differing protocols, the fact that they yielded similar results to those found in Table 1 suggests that DNA-DNA hybridization studies and whole-genome alignments are measuring the same quantity, i.e. sequence identity, by compatible methods.

These data allow us to estimate how well one factor involved in genome divergence - random mutation within vertically transferred genetic material - correlates with total divergence between pairs of genomes. Univariate regression analysis of whole-genome sequence identity with respect to conserved-region sequence identity (Fig. 1a) showed an excellent fit to the linear model $\left(P<0 \cdot 001, R^{2}=0 \cdot 871\right)$. The 
Table 1. DNA sequence comparisons for related bacterial genomes

'Grouping' indicates the genera (or in the case of Escherichia, Shigella and Salmonella, the closely related genera) used to group the sequences for alignment studies. Genomes within each group were aligned in pairwise fashion by using the NUCMER application, as described in Methods. 'Conserved-region' and 'whole-genome' DNA sequence identity scores are described in the text.

\begin{tabular}{|c|c|c|c|}
\hline \multirow[t]{2}{*}{ Grouping and genome comparison } & & \multicolumn{2}{|c|}{ DNA sequence identity } \\
\hline & & Conserved regions & Whole genomes \\
\hline \multicolumn{4}{|l|}{ Bacillus } \\
\hline Bacillus anthracis A2012 & Bacillus halodurans C-125 & $0 \cdot 742$ & $0 \cdot 039$ \\
\hline Bacillus anthracis A2012 & Bacillus subtilis 168 & $0 \cdot 747$ & $0 \cdot 057$ \\
\hline Bacillus halodurans C-125 & Bacillus subtilis 168 & $0 \cdot 769$ & $0 \cdot 052$ \\
\hline \multicolumn{4}{|l|}{ Buchnera } \\
\hline Buchnera aphidicola $\mathrm{Sg}^{\mathrm{T}}$ & Buchnera sp. APS & $0 \cdot 780$ & $0 \cdot 705$ \\
\hline \multicolumn{4}{|l|}{ Chlamydia } \\
\hline Chlamydia muridarum $\mathrm{MoPn}^{\mathrm{T}}$ & Chlamydia trachomatis D/UW-3/CX & $0 \cdot 809$ & $0 \cdot 736$ \\
\hline \multicolumn{4}{|l|}{ Chlamydophila } \\
\hline Chlamydophila pneumoniae AR39 & Chlamydophila pneumoniae CWL029 & $0 \cdot 999$ & $0 \cdot 999$ \\
\hline Chlamydophila pneumoniae AR39 & Chlamydophila pneumoniae J138 & $0 \cdot 999$ & $0 \cdot 999$ \\
\hline Chlamydophila pneumoniae CWL029 & Chlamydophila pneumoniae J138 & $0 \cdot 999$ & $0 \cdot 999$ \\
\hline \multicolumn{4}{|l|}{ Clostridium } \\
\hline Clostridium acetobutylicum ATCC $824^{\mathrm{T}}$ & Clostridium perfringens 13 & $0 \cdot 754$ & $0 \cdot 086$ \\
\hline \multicolumn{4}{|l|}{ Escherichia-like } \\
\hline Escherichia coli K-12 MG1655 & Escherichia coli O157:H7 EDL933 & $0 \cdot 977$ & $0 \cdot 812$ \\
\hline Escherichia coli K-12 MG1655 & Salmonella typhi CT18 & $0 \cdot 809$ & $0 \cdot 534$ \\
\hline Escherichia coli K-12 MG1655 & Salmonella typhimurium LT2 & $0 \cdot 809$ & $0 \cdot 537$ \\
\hline Escherichia coli $\mathrm{K}-12 \mathrm{MG} 1655$ & Shigella flexneri 2a 301 & $0 \cdot 978$ & $0 \cdot 859$ \\
\hline Escherichia coli O157: H7 EDL933 & Salmonella typhi CT18 & $0 \cdot 808$ & $0 \cdot 490$ \\
\hline Escherichia coli O157: H7 EDL933 & Salmonella typhimurium LT2 & $0 \cdot 807$ & $0 \cdot 500$ \\
\hline Escherichia coli O157: H7 EDL933 & Shigella flexneri 2a 301 & $0 \cdot 975$ & $0 \cdot 795$ \\
\hline Salmonella typhi CT18 & Salmonella typhimurium LT2 & $0 \cdot 982$ & $0 \cdot 883$ \\
\hline Salmonella typhi CT18 & Shigella flexneri 2a 301 & $0 \cdot 813$ & $0 \cdot 536$ \\
\hline Salmonella typhimurium LT2 & Shigella flexneri 2a 301 & $0 \cdot 809$ & $0 \cdot 520$ \\
\hline \multicolumn{4}{|l|}{ Helicobacter } \\
\hline Helicobacter pylori 26695 & Helicobacter pylori $\mathrm{J} 99$ & $0 \cdot 930$ & $0 \cdot 876$ \\
\hline \multicolumn{4}{|l|}{ Listeria } \\
\hline Listeria innocua CLIP 11262 & Listeria monocytogenes EGD-e & $0 \cdot 882$ & $0 \cdot 779$ \\
\hline \multicolumn{4}{|l|}{ Mycobacterium } \\
\hline Mycobacterium leprae & Mycobacterium tuberculosis CDC1551 & $0 \cdot 777$ & $0 \cdot 360$ \\
\hline Mycobacterium leprae & Mycobacterium tuberculosis $\mathrm{H} 37 \mathrm{Rv}$ & $0 \cdot 777$ & $0 \cdot 362$ \\
\hline Mycobacterium tuberculosis CDC1551 & Mycobacterium tuberculosis $\mathrm{H} 37 \mathrm{Rv}$ & $0 \cdot 998$ & $0 \cdot 993$ \\
\hline \multicolumn{4}{|l|}{ Mycoplasma } \\
\hline Mycoplasma genitalium $\mathrm{G}-37^{\mathrm{T}}$ & Mycoplasma pneumoniae ATCC 29342 & $0 \cdot 749$ & $0 \cdot 253$ \\
\hline Mycoplasma genitalium $\mathrm{G}-37^{\mathrm{T}}$ & Mycoplasma pulmonis UAB CT & $0 \cdot 725$ & $0 \cdot 018$ \\
\hline Mycoplasma pneumoniae ATCC 29342 & Mycoplasma pulmonis $\mathrm{UAB} \mathrm{CT}$ & $0 \cdot 743$ & $0 \cdot 012$ \\
\hline \multicolumn{4}{|l|}{ Neisseria } \\
\hline Neisseria meningitidis MC58 & Neisseria meningitidis Z2491 & $0 \cdot 957$ & $0 \cdot 908$ \\
\hline \multicolumn{4}{|l|}{ Rickettsia } \\
\hline Rickettsia conorii NIAID Malish $7^{\mathrm{T}}$ & Rickettsia prowazekii Madrid E & $0 \cdot 847$ & $0 \cdot 654$ \\
\hline \multicolumn{4}{|l|}{ Staphylococcus } \\
\hline Staphylococcus aureus Mu50 & Staphylococcus aureus MW2 & $0 \cdot 984$ & $0 \cdot 922$ \\
\hline Staphylococcus aureus Mu50 & Staphylococcus aureus N315 & $0 \cdot 999$ & $0 \cdot 981$ \\
\hline Staphylococcus aureus MW2 & Staphylococcus aureus N315 & $0 \cdot 985$ & $0 \cdot 933$ \\
\hline \multicolumn{4}{|l|}{ Streptococcus } \\
\hline Streptococcus agalactiae 2603V/R & Streptococcus pneumoniae R6 & $0 \cdot 787$ & $0 \cdot 164$ \\
\hline Streptococcus agalactiae 2603V/R & Streptococcus mutans UA159 & $0 \cdot 768$ & $0 \cdot 239$ \\
\hline Streptococcus agalactiae 2603V/R & Streptococcus pyogenes M1 GAS SF370 & $0 \cdot 800$ & $0 \cdot 296$ \\
\hline
\end{tabular}


Table 1. cont.

Grouping and genome comparison

DNA sequence identity

\begin{tabular}{|c|c|c|c|}
\hline & & \\
\hline & & Conserved regions & Whole genomes \\
\hline Streptococcus agalactiae 2603V/R & Streptococcus pyogenes MGAS315 & $0 \cdot 794$ & $0 \cdot 278$ \\
\hline Streptococcus agalactiae $2603 \mathrm{~V} / \mathrm{R}$ & Streptococcus pyogenes MGAS 8232 & $0 \cdot 794$ & $0 \cdot 279$ \\
\hline Streptococcus pneumoniae R6 & Streptococcus mutans UA159 & $0 \cdot 769$ & $0 \cdot 188$ \\
\hline Streptococcus pneumoniae R6 & Streptococcus pyogenes M1 GAS SF370 & $0 \cdot 780$ & $0 \cdot 186$ \\
\hline Streptococcus pneumoniae R6 & Streptococcus pyogenes MGAS315 & $0 \cdot 779$ & $0 \cdot 185$ \\
\hline Streptococcus pneumoniae R6 & Streptococcus pyogenes MGAS8232 & $0 \cdot 779$ & $0 \cdot 189$ \\
\hline Streptococcus mutans UA159 & Streptococcus pyogenes M1 GAS SF370 & $0 \cdot 765$ & $0 \cdot 232$ \\
\hline Streptococcus mutans UA159 & Streptococcus pyogenes MGAS315 & $0 \cdot 761$ & $0 \cdot 222$ \\
\hline Streptococcus mutans UA159 & Streptococcus pyogenes MGAS8232 & $0 \cdot 766$ & $0 \cdot 226$ \\
\hline Streptococcus pyogenes M1 GAS SF370 & Streptococcus pyogenes MGAS315 & $0 \cdot 984$ & $0 \cdot 904$ \\
\hline Streptococcus pyogenes M1 GAS SF370 & Streptococcus pyogenes MGAS8232 & $0 \cdot 982$ & $0 \cdot 914$ \\
\hline Streptococcus pyogenes MGAS315 & Streptococcus pyogenes MGAS 8232 & $0 \cdot 982$ & $0 \cdot 925$ \\
\hline \multicolumn{4}{|l|}{ Xanthomonas } \\
\hline Xanthomonas axonopodis 306 & Xanthomonas campestris ATCC $33913^{\mathrm{T}}$ & $0 \cdot 844$ & $0 \cdot 523$ \\
\hline \multicolumn{4}{|l|}{ Yersinia } \\
\hline Yersinia pestis $\mathrm{CO} 92$ & Yersinia pestis KIM & $0 \cdot 999$ & $0 \cdot 997$ \\
\hline
\end{tabular}
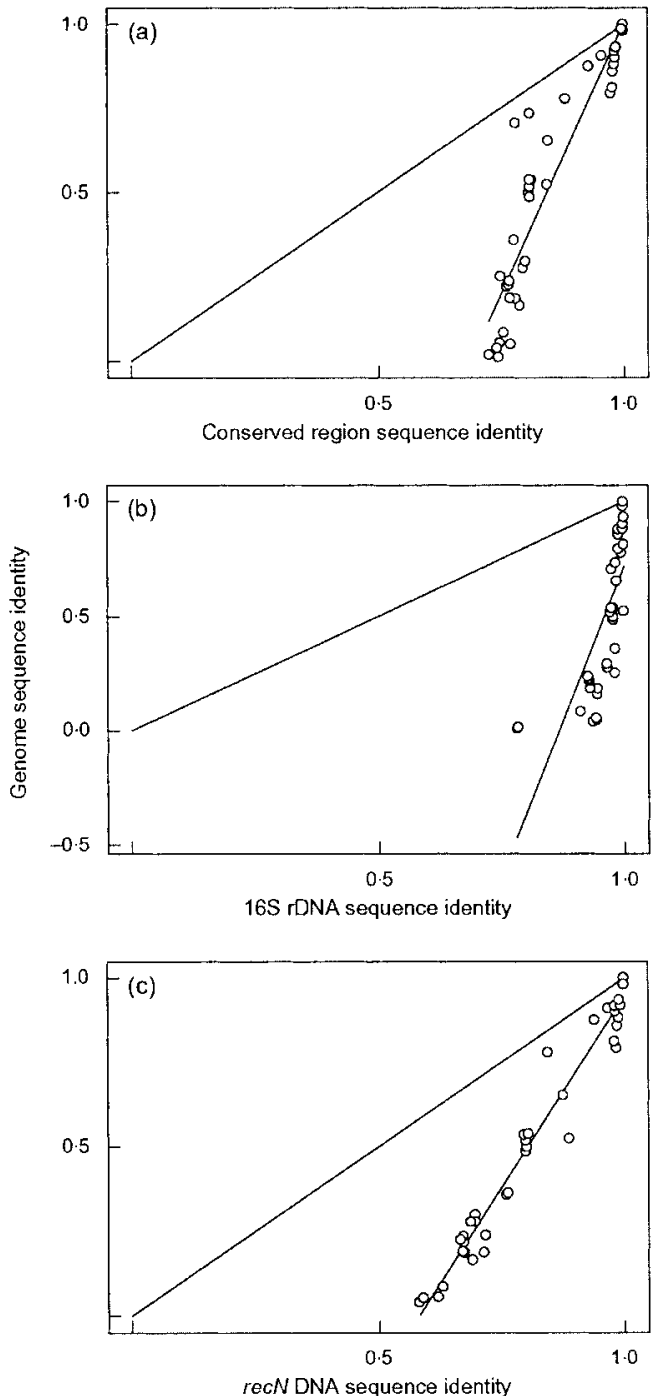

simplest interpretation of this result is that during bacterial speciation, the various forces that change genome sequence content act at discrete rates in a time-dependent manner. As a result, the ratio between sequence differences in conserved regions and overall genome sequence differences remains fairly constant, at least while the bacteria are related as closely as those analysed here. This interpretation, if true, means that the proposal of Stackebrandt et al. (2002) is quite reasonable: a rational definition of bacterial species could be based on sequence analysis of a set of conserved genes. If the relatedness of whole genomes can be measured by examining the subset of genes they share, then a small but representative set of shared genes should successfully predict genome relatedness.

\section{DNA sequence identity between individual gene orthologues}

Candidate genes for a 'species prediction set' were identified from these bacterial genomes by applying four criteria that were consistent with the overall goal of this study: to develop a practical tool for bacterial taxonomy. First, the genes must be widely distributed among genomes, with orthologous sequences appearing in most, if not all, freeliving bacteria. Secondly, because the occurrence of gene families could make sequencing and alignment technically difficult, each of the 'prediction set' genes must be unique within a given genome, without close paralogues that could confuse analysis. Thirdly, individual gene sequences must

Fig. 1. Linear regression analysis of whole-genome sequence identity versus sequence identities of (a) conserved regions; (b) 16S rDNA sequences; and (c) recN sequences. Lines indicate the calculated best fit for the data and the hypothetical fit if the slope of the regression line was equal to 1 . 
be long enough to contain phylogenetically useful information but short enough to be sequenced economically with a small set of primers. Finally, the sequences must predict whole-genome relationships with acceptable precision and accuracy. The first criterion was satisfied by preliminary genome comparisons among the bacteria listed in Table 1 , which yielded a pool of over 100 candidate genes (not shown). BLAST searches eliminated candidates that had close paralogues within one or more of the genomes. Sequences that encoded merely hypothetical proteins were also struck from the list, as were those that were deemed to be shorter
( $<900$ bp) or longer ( $>2250 \mathrm{bp}$ ) than optimum. The 32 candidate genes that remained after application of these criteria are listed in Table 2. The $16 \mathrm{~S}$ rRNA gene, although it does not encode a protein, was added to the list of candidates because of its wide usage in taxonomic studies.

It remained to be determined which of the candidate genes best satisfied the final criterion by predicting wholegenome relationships. For each gene listed in Table 2, orthologous sequences from each of the genomes were divided into genus groups (listed in Table 1). Sequences

Table 2. Single-variable regression analysis for whole-genome sequence identity versus individual gene sequence identity

Gene names used for orthologous sequences are consistently those used in the E. coli genome annotation. Gene nomenclature may vary among bacterial species. Orthologous sequences are found in each of the genomes analysed in this study, except as listed under 'Exceptions'. Abbreviations: BAC-ANTH, Bacillus anthracis; BUC, Buchnera; CHA, Chlamydia; CHO, Chlamydophila; MYB, Mycobacterium; MYP, Mycoplasma; MYP-PULM, Mycoplasma pulmonis; NEI, Neisseria; RIC, Rickettsia; STA, Staphylococcus; STR, Streptococcus; STR-8232, Streptococcus pyogenes MGAS8232.

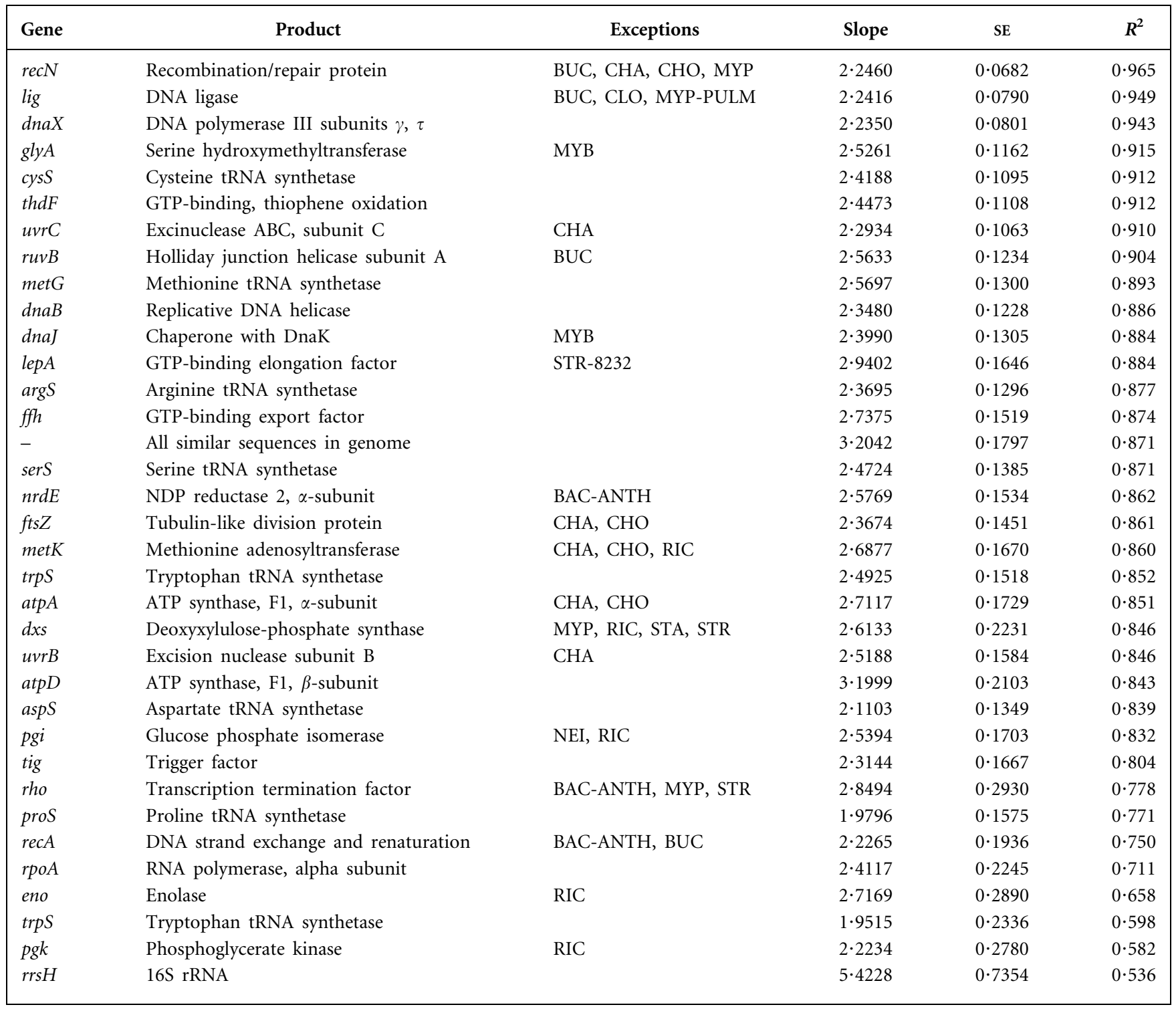


within each group were analysed following multiple alignment to compute a distance matrix that quantified sequence identity. Therefore, for each genome comparison, there was a sequence identity score for the whole genomes as well as 33 identity scores for individual genes. A spreadsheet listing sequence identity scores for all candidates with each genome comparison is available as supplementary data in IJSEM Online. These sequence identity scores were analysed by linear regression to determine which gene comparison could best predict genome relatedness.

For each gene, the plot of genome sequence identity versus individual gene sequence identity fit a linear model with $P<0 \cdot 01$. Goodness of fit varied widely among the candidate genes, with $R^{2}$ values ranging from 0.536 to 0.965 . Eight of the genes had an $R^{2}$ value of 0.9 or better, making them outstanding candidates for a 'species prediction' sequence set (Table 2). The individual candidate gene with the poorest ability to predict genome relatedness on a genus or species level was $16 \mathrm{~S}$ rDNA, the gene that encodes $16 \mathrm{~S}$ rRNA (Fig. 1b); as others have noted (Fox et al., 1992; Stackebrandt \& Goebel, 1994), it is simply too highly conserved to differentiate reliably between closely related taxa. The candidate gene with the greatest potential for predicting genome relatedness at the genus or subgenus level was $\operatorname{recN}$ (Fig. 1c), a recombination and repair protein-encoding gene that is found in each of the freeliving bacterial genomes analysed, as well as in the two Rickettsia species. Among genes found in every bacterial genome analysed, the highest-scoring candidate was dnaX, a gene that encodes two subunits of DNA polymerase III. Whilst $r e c N$ is slightly superior to $d n a X$ in terms of fit to whole-genome data $\left(R^{2}, 0.965\right.$ and 0.943 , respectively), the latter sequence may prove to be particularly useful in analysis of taxa characterized by genome reduction.

A parallel study analysed amino acid sequence identities of the predicted products for each of the candidate genes (not shown). In each case, the gene sequences showed a better fit to genome relatedness than the predicted protein sequences. Whilst protein sequences have often been analysed to compare distantly related organisms or ancient gene duplications (Brown et al., 2001; Delcher et al., 2002), DNA sequences may be more useful for distinguishing close phylogenetic relationships.

This 'bacterial species prediction' set differs from sequence sets that were assembled for the purpose of constructing universal phylogenetic trees (Brown et al., 2001). The purpose of those studies is to detect relationships among very distantly related organisms at the domain level, whereas the purpose of the current study is to distinguish between closely related organisms at the species level. Many of the highest scoring sequences in Table 2 have no known orthologues outside bacteria and so cannot be used to construct universal trees. Further, the practical aims of the current study impose selection criteria that would be unnecessary in a universal tree study, such as moderate gene length and absence of close paralogues. Nevertheless, there is some overlap, including certain tRNA synthetases and DNA and RNA polymerase subunits, between the bacterial 'species prediction' set developed in this study and the 'universal tree' set of Brown et al. (2001).

\section{Predictive models}

Linear regression analysis yielded simple predictive models for high-scoring candidates. Genome relatedness for two related bacterial strains can be predicted by the following formula:

$S I_{\text {genome }}=-1 \cdot 30+2 \cdot 25\left(S I_{\text {rec } N}\right)$

where $S I_{\text {genome }}$ is the predicted DNA sequence identity shared by the genomes and $S I_{r e c N}$ is the sequence identity shared by their $\operatorname{recN}$ orthologues. The actual genome sequence identity is plotted against the $r e c N$ prediction in Fig. 2(a) for each of the genomes listed in Table 1. The
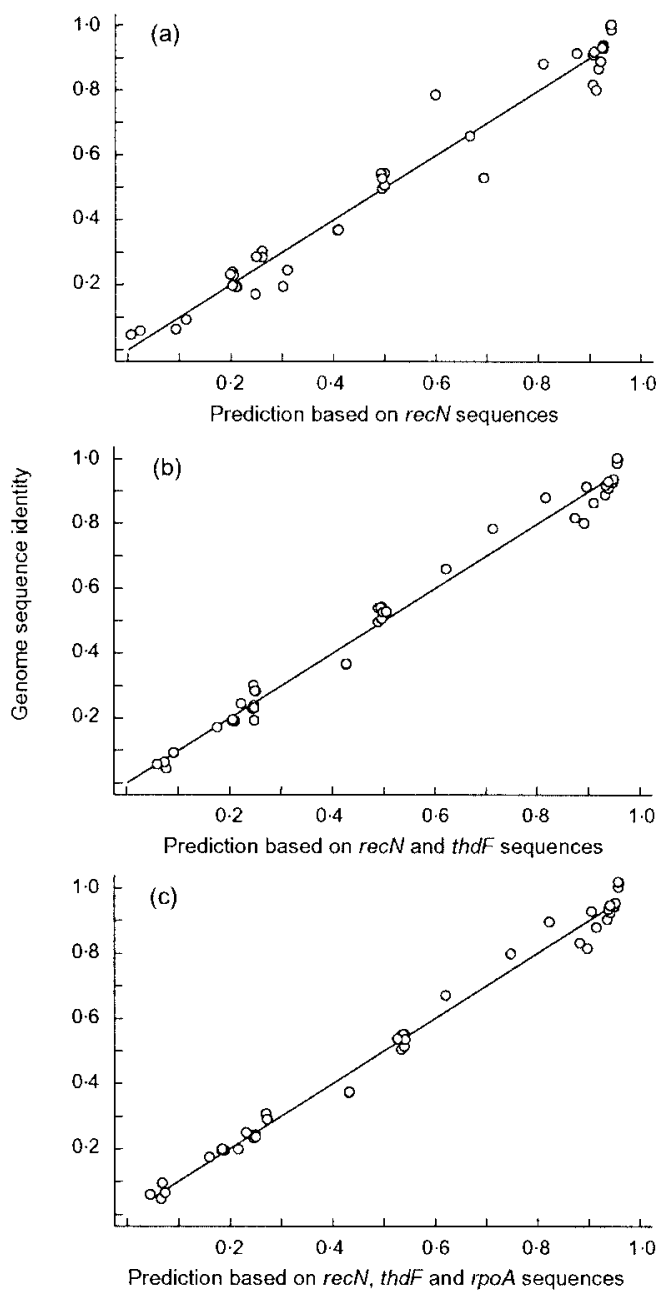

Fig. 2. Linear regression analysis of whole-genome sequence identity scores versus scores predicted based on the models described in the text for (a) $\operatorname{rec} N$; (b) $\operatorname{rec} N$ and thdF; and (c) recN, thd $F$ and rpoA. 
prediction is surprisingly powerful: it fits a linear model with $P<0.001$ and $R^{2}=0.965$. The mean absolute residual (i.e. the mean difference between the predicted and actual genome sequence identity) is only $0 \cdot 044$. Similar results (not shown) were obtained by using $d n a X$ sequences with the following formula:

$S I_{\text {genome }}=-1 \cdot 29+2 \cdot 24\left(S I_{\text {dnaX }}\right)$

A $95 \%$ prediction interval can be obtained from the recN prediction formula above. Based on these results, one can make the following conclusions: if the recN DNA sequences for two bacterial strains or isolates are $<84 \%$ similar, we can be $95 \%$ confident that their genome sequences are $<70 \%$ similar and that the bacteria belong to different species. If the recN DNA sequences are $>96 \%$ similar, then by the same reasoning, we can be $95 \%$ confident that the bacteria belong to the same species. If the $r e c N$ sequences are between 84 and $96 \%$ similar, it is questionable whether the genome sequence identity is greater than or less than $70 \%$, making the species identity for these strains uncertain.

Step-wise linear regression procedures were used to determine whether inclusion of sequence data from other candidate genes improved the predicting power of $\operatorname{recN}$. The results suggest that the best two-gene combination (that is, the combination with the highest $R^{2}$ value) was recN and $t h d F$, which encodes a thiophene oxidation enzyme (Fig. 2b), whereas the best three-gene combination was $r e c N$, thdF and rpoA, which encodes the RNA polymerase $\alpha$-subunit (Fig. 2c). Interestingly, the best sets used a combination of genes that were highly $(r p o A)$, moderately $(t h d F)$ and weakly $(r e c N)$ conserved. Prediction models resulting from this analysis were:

$$
\begin{aligned}
& S I_{\text {genome }}=-1 \cdot 67+0 \cdot 707\left(S I_{r e c N}\right)+1 \cdot 92\left(S I_{\text {thdF }}\right) \\
& S I_{\text {genome }}=-1 \cdot 88+0 \cdot 52\left(S I_{r e c N}\right)+1 \cdot 78\left(S I_{t h d F}\right)+0 \cdot 52\left(S I_{r p o A}\right)
\end{aligned}
$$

where $S I_{\text {genome }}$ is the predicted DNA sequence identity shared by the genomes and $S I_{r e c N}, S I_{t h d F}$ and $S I_{r p o A}$ are the sequence identities shared by the recN, thdF and rpoA orthologues, respectively. For the two- and three-gene models, $R^{2}$ values improved to 0.986 and 0.989 , respectively, and the mean absolute residuals improved to 0.032 and $0 \cdot 026$, respectively.

Bacterial genome sequences continue to become available in increasing numbers. Following the statistical modelling phase of this study, public databases were re-examined for new whole-genome sequences that either fit within the genus groups analysed before or defined new groups. Several new comparisons were possible, serving as a test of the validity of the predictive models (Table 3 ). The genomes were analysed as before and for each genome pair, sequence identity scores were computed for whole genomes and for the predictor genes $r e c N$, thdF and rpoA. The single-, twoand three-gene sets predicted whole-genome sequence identity with mean residual values of $0.049,0.032$ and $0 \cdot 040$, respectively. This second test set of comparisons yielded results that were completely in harmony with those of the first set of comparisons.

Table 3. Predictions of DNA sequence identity for pairs of genomes

\begin{tabular}{|c|c|c|c|c|c|c|c|c|}
\hline \multicolumn{2}{|c|}{ Genome comparison } & \multicolumn{4}{|c|}{ DNA sequence identity } & \multicolumn{3}{|c|}{ Genome prediction } \\
\hline Corynebacterium diphtheriae & Corynebacterium glutamicum & $0 \cdot 591$ & $0 \cdot 847$ & - & $0 \cdot 126$ & $0 \cdot 030$ & - & - \\
\hline Corynebacterium efficiens & Corynebacterium glutamicum & $0 \cdot 735$ & $0 \cdot 872$ & - & $0 \cdot 336$ & $0 \cdot 354$ & - & - \\
\hline Escherichia coli CFT073 & Escherichia coli $\mathrm{K}-12$ & $0 \cdot 969$ & $1 \cdot 000$ & $0 \cdot 950$ & $0 \cdot 790$ & $0 \cdot 880$ & $0 \cdot 839$ & $0 \cdot 835$ \\
\hline Escherichia coli CFT073 & Salmonella typhimurium & $0 \cdot 803$ & $0 \cdot 976$ & $0 \cdot 838$ & $0 \cdot 511$ & $0 \cdot 507$ & $0 \cdot 507$ & $0 \cdot 537$ \\
\hline Escherichia coli CFT073 & Salmonella flexneri & $0 \cdot 969$ & $0 \cdot 997$ & $0 \cdot 953$ & $0 \cdot 752$ & $0 \cdot 880$ & $0 \cdot 845$ & $0 \cdot 839$ \\
\hline Mycobacterium bovis & Mycobacterium tuberculosis CDC & $0 \cdot 999$ & $1 \cdot 000$ & $1 \cdot 000$ & $0 \cdot 991$ & $0 \cdot 948$ & $0 \cdot 956$ & $0 \cdot 939$ \\
\hline Mycobacterium bovis & Mycobacterium leprae & $0 \cdot 758$ & $0 \cdot 883$ & $0 \cdot 811$ & $0 \cdot 366$ & $0 \cdot 406$ & $0 \cdot 423$ & $0 \cdot 417$ \\
\hline Neisseria meningitidis FAM & Neisseria gonorrhoeae & $0 \cdot 954$ & $0 \cdot 993$ & $0 \cdot 952$ & $0 \cdot 804$ & $0 \cdot 847$ & $0 \cdot 832$ & $0 \cdot 827$ \\
\hline Staphylococcus epidermidis & Staphylococcus aureus MU50 & $0 \cdot 775$ & $0 \cdot 945$ & $0 \cdot 805$ & $0 \cdot 406$ & $0 \cdot 444$ & $0 \cdot 424$ & $0 \cdot 447$ \\
\hline Staphylococcus epidermidis & Staphylococcus aureus MW2 & $0 \cdot 775$ & $0 \cdot 945$ & $0 \cdot 807$ & $0 \cdot 409$ & $0 \cdot 444$ & $0 \cdot 427$ & $0 \cdot 451$ \\
\hline Staphylococcus epidermidis & Staphylococcus aureus N315 & $0 \cdot 775$ & $0 \cdot 945$ & $0 \cdot 806$ & $0 \cdot 412$ & $0 \cdot 444$ & $0 \cdot 425$ & $0 \cdot 449$ \\
\hline Yersinia enterocolitica & Yersinia pestis KIM & $0 \cdot 822$ & $0 \cdot 985$ & $0 \cdot 851$ & $0 \cdot 523$ & $0 \cdot 550$ & $0 \cdot 545$ & $0 \cdot 574$ \\
\hline
\end{tabular}

Predictions 1, 2 and 3 are based on the single-, two- and three-gene prediction models that are described in the text. 
In conclusion, the hypothesis that sequences from proteinencoding genes can predict genome relatedness accurately is supported strongly by these studies. Before conducting full-scale studies based on these concepts, however, researchers should keep at least two cautions in mind. First, these results are based on a limited number of bacterial species; as additional genome data become available, the statistical models presented in this paper will doubtless require refinement. Secondly, it is certainly possible that within a given genus, phylogenies constructed by using a small number of genes can be perturbed radically if horizontal transfer has occurred among those genes. Nevertheless, the results in this paper demonstrate that analysis of even a single carefully selected gene, such as $\operatorname{rec} N$ or $d n a X$, could be a powerful tool for discriminating between species represented among a large group of bacterial isolates. Clearly a set of 'species predictor' genes has superior precision when compared to a single predictor gene, but there is a diminishing degree of improvement as each new sequence is added. The five-gene set envisioned by Stackebrandt et al. (2002) may be more genes than are actually required to equal or even surpass the power of DNA-DNA reassociation in assigning related bacterial isolates to species.

\section{ACKNOWLEDGEMENTS}

The author thanks T. Davidsen and A. Phillippy for helpful advice on using the NUCMER package, S. Shew and M. Abdullah for technical assistance in software implementation and S. Hoshaw-Woodard for performing the statistical analyses reported in this paper.

\section{REFERENCES}

Brenner, D. J., Fanning, G. R., Johnson, K. E., Citarella, R. V. \& Falkow, S. (1969). Polynucleotide sequence relationships among members of Enterobacteriaceae. J Bacteriol 98, 637-650.

Brenner, D. J., Fanning, G. R., Skerman, F. J. \& Falkow, S. (1972). Polynucleotide sequence divergence among strains of Escherichia coli and closely related organisms. J Bacteriol 109, 933-965.
Brown, J. R., Douady, C. J., Italia, M. J., Marshall, W. E. \& Stanhope, M. J. (2001). Universal trees based on large combined protein sequence data sets. Nat Genet 28, 281-285.

Crosa, J. H., Brenner, D. J., Ewing, W. H. \& Falkow, S. (1973). Molecular relationships among the Salmonelleae. J Bacteriol 115, 307-315.

Delcher, A. L., Phillippy, A., Carlton, J. \& Salzberg, S. L. (2002). Fast algorithms for large-scale genome alignment and comparison. Nucleic Acids Res 30, 2478-2483.

Fox, G. E., Wisotzkey, J. D. \& Jurtshuk, P., Jr (1992). How close is close: $16 \mathrm{~S}$ rRNA sequence identity may not be sufficient to guarantee species identity. Int J Syst Bacteriol 42, 166-170.

Fukushi, H. \& Hirai, K. (1989). Genetic diversity of avian and mammalian Chlamydia psittaci strains and relation to host origin. J Bacteriol 171, 2850-2855.

Gürtler, V. \& Mayall, B. C. (2001). Genomic approaches to typing, taxonomy and evolution of bacterial isolates. Int J Syst Evol Microbiol 51, 3-16.

Johnson, J. L. (1994). Similarity analysis of DNAs. In Methods for General and Molecular Bacteriology, pp. 655-682. Edited by P. Gerhardt, R. G. E. Murray, W. A. Wood \& N. R. Krieg. Washington, DC: American Society for Microbiology.

Rosselló-Mora, R. \& Amann, R. (2001). The species concept for prokaryotes. FEMS Microbiol Rev 25, 39-67.

Somerville, H. J. \& Jones, M. L. (1972). DNA competition studies within the Bacillus cereus group of bacilli. J Gen Microbiol 73, 257-265.

Stackebrandt, E. \& Goebel, B. M. (1994). Taxonomic note: a place for DNA-DNA reassociation and 16S rRNA sequence analysis in the present species definition in bacteriology. Int J Syst Bacteriol 44, 846-849.

Stackebrandt, E., Frederiksen, W., Garrity, G. M. \& 10 other authors (2002). Report of the ad hoc committee for the re-evaluation of the species definition in bacteriology. Int J Syst Evol Microbiol 52, 1043-1047.

Thompson, J. D., Higgins, D. G. \& Gibson, T. J. (1994). CLUSTAL W: improving the sensitivity of progressive multiple sequence alignment through sequence weighting, position-specific gap penalties and weight matrix choice. Nucleic Acids Res 22, 4673-4680.

Wayne, L. G., Brenner, D. J., Colwell, R. R. \& 9 other authors (1987). International Committee on Systematic Bacteriology. Report of the ad hoc committee on reconciliation of approaches to bacterial systematics. Int J Syst Bacteriol 37, 463-464.

Weiss, E., Schramek, S., Wilson, N. N. \& Newman, L. W. (1970). Deoxyribonucleic acid heterogeneity between human and murine strains of Chlamydia trachomatis. Infect Immun 2, 24-28. 